\title{
Need for Specialized Therapeutic Stem Cells Banks Equipped with Tumor Regression Enzymes and Anti-Tumor Genes
}

Mujib Ullah*

Interventional Regenerative Medicine and Imaging Lab, Department of Radiology, School of Medicine, Stanford University, California, USA.

${ }^{*}$ Corresponding Author:

Mujib Ullah, Dr. Medic, PhD

Department of Radiology, Stanford University

3155 Porter Drive, Palo Alto, CA, 94304, USA

E-mail: ullah@stanford.edu

\begin{abstract}
Stem cells are currently being used in many clinical trials for regenerative purposes. These are promising results for stem cells in the treatment of several diseases, including cancer. Nevertheless, there are still many variables which should be addressed before the application of stem cells for cancer treatment. One approach should be to establish well-characterized therapeutic stem cell banks to minimize the variation in results from different clinical trials and facilitate their effective use in basic and translational research.
\end{abstract}

\section{Keywords:}

Stem cells banks, Cancer, Cytosine deaminase, Tumor, Gene therapy 


\section{Introduction}

Cancer is the leading cause of deaths worldwide [1, 2]. One of the most innovative approaches to treat cancer is suicide gene therapy [3, 4]. Cytosine Deaminase (CD) is one of many suicide genes used in cancer gene therapy to sensitize tumor cells to chemotherapy $[3,5]$. CD encodes an enzyme that can convert non-toxic 5-flurocytosine to toxic 5-fluorouracil (5-FU) [3,6]. 5-FU is a potent chemotherapeutic drug and has significant anticancer effects for regression of tumors [3,5]. One limitation of previous reports was to engineer CD gene into viruses or mesenchymal stem cells (MSCs) $[5,7$, 8]. Viruses are protumor in some cases and are relatively poor in targeting tumor cells [5, $7,9]$. The use of viruses can also pose a risk to patients in a variety of ways by triggering immune and inflammatory reactions [5, 7, 9]. Furthermore, viruses cannot distinguish between normal and cancer cells and have the capability to self-recover and cause diseases once inside the body [5, 7, 9]. On the other hand, MSCs offer great therapeutic promise to treat cancer patients [10-12]. However, the clinical trials of MSCs are hindered by many factors like considerable donor variations, epigenetic reprogramming, senescence in culture, protumor potential of MSCs under certain conditions, limited expandability, and difficulty for transgene expression [11-15]. Additionally, the epitopes to define MSCs are still not very specific, and differentiation assays are difficult to standardize [12, 13, 16-18]. One solution may be to establish huge repositories of standardized iPSCs-derived MSCs banks for worldwide distribution that can be shared with scientists and clinicians across the globe [19]. The insertion of therapeutic genes into these iPSCs can make them more tumor precision-based banks [3,5]. The use of iPSCsderived MSCs has an advantage over bone marrow-derived MSCs, as they grow in less time and propagate more, undergo more population doublings, experience less growtharrest in culture, and have better healing abilities [3, 12, 13]. Importantly, they did not form teratomas, and they are less impacted to genetic drift and mutations $[12,13]$.

The iPSCs-MSCs have strong tropism toward cancer cells and can serve as cellular vehicles to deliver $C D$ within or near the tumor sites $[6,13]$. The beauty of this approach is that iPSCs-MSCs with CD itself can undergo cell death caused by suicide effects of 5FU, ensuring the long-term safety of this method $[3,5]$. After suicide action of these cells, there is no leftover gene $[3,5]$. Thus, it eliminates the concerns like insertional mutagenesis, gene activation in long-term effects commonly associated with such therapies $[5,13]$.

In case of challenging brain tumors, iPSCs-MSCs could be designed to treat the remaining tumor masses after surgery to minimize tumor reoccurrence chances in patients $[5,20]$. After administration of this therapy, once the prodrug starts destruction of the cancerous cells, this process may also prompt the immune system to attack the tumor cells $[5,20]$. This strategy can be effective to treat recurrent cancer patients who received a standard treatment (surgery, radiotherapy, and chemotherapy).

These therapeutic cells can be freshly prepared from standardized master banks and can be given to patients who qualify for such kind of therapy [21]. However, the master cell 
bank must be produced in a good manufacturing practice-compliant facility and should be filed to the Food and Drug Administration to assess toxicity and safety trials [21]. A safe and efficient gene carrier is a prerequisite for successful anticancer therapy, and we believe that iPSCs-MSCs can be a suitable cargo for CD enzyme $[3,5]$. Whether or not iPSCs-MSCs with CD gene therapy make it to market is currently unclear.

\section{Source of stem cells for therapeutic banks}

There are several sources of stem cells, but bone marrow and adipose tissue is the bestknown source for several types of cells $[17,22,23]$. Different types of stem cells are totipotent stem cells, pluripotent stem cells, adult stem cells, umbilical cord-derived stem cells, embryonic stem cells, and reprogrammed stem cells [15, 17, 23]. Recent data indicates that adipose-derived stem cells (ASCs) and bone marrow-derived stem cells (BMSCs) share many biological features; however, there are some variations in their surface epitopes, immunophenotype, differentiation potential, cytokine secretion profile, proteomic profile, and their ability to secrete growth factors [24, 25]. BMSCs and ASCs populations differ in heterogeneity, potency, doubling time, and proliferation, according to conflicting results in clinical trials [24, 25]. Most importantly, these differences support the use of induced pluripotent stem cells because they form a more uniform population and, according to clinical results, seem to be more effective $[25,26]$. Many studies have shown the biology, regeneration ability, and clinical applications of iPSCs in detail [27-30]. Banked iPSCs are not only used for research but are also commonly used for clinical applications such as transplantations and the field of regenerative medicine [30]. The iPSCs that have been studied for regenerative medicine have been extensively investigated for their therapeutic potential [30]. One special feature of these cells is that they can easily produce millions and even billions of cells to prepare large banks [27, 30].

Therefore, this necessitates the establishment of a major research project to globally bank the iPSCs and to characterize them more fully with the latest techniques and instruments [31]. One approach to this can be to insert therapeutic genes for cancer prevention or diagnosis or otherwise to "pre-activate" them so that they become more powerful in healing tissues and killing cancer cells [31]. One method to derive these immortal iPSCs cells is to obtain them from essentially any tissue in adult donors [31]. One advantage of deriving iPSCs this way is that they can be used to generate an essentially unlimited supply of thoroughly standardized cells while excluding the tendency of normal iPSCs to form tumors which then presents a danger in using iPSCs in patients [26, 32]. Another advantage is that the exosomes that iPSCs produce appear to have even greater therapeutic effects than the iPSCs themselves [8, 33]. Because of these two advantages, iPSCs banks would have two strategies to treat diseases: one based on the administration of iPSCs to patients and the other based on the administration of the proteins or exosomes that iPSCs produce [26, 32].

Therefore, one can confidently state that both iPSCs themselves and their derivatives such as their exosomes are potent instruments and a great choice for banks and for later 
use in biomedicine, cell replacement therapy, and cancer prevention, furthering the need for the maximum standardization and safety [25, 34].

\section{Selection of anti-tumor genes for banks}

Among various treatment strategies, suicide gene therapy attracts special attention because it allows for selective conversion of non-toxic compounds into cytotoxic drugs that selectively kill the cancer cells $[5,8]$. There are many suicide genes such as cytosine deaminase, the herpes simplex virus, cytochrome P450, thymidine kinase, Purine Nucleoside Phosphorylase, carboxypeptidase G2, nitro-reductase and many more [5, 34]. The cytosine deaminase enzyme, specifically, effectively kills cancer cells by converting 5-FC into toxic 5-FU, which is shown to easily diffuse into cancer cells to enhance apoptosis, and accelerate protein starvation of tumor cells, forcing them into cellular death $[35,36]$. Additionally, the drug 5-FC does not need a gap junction to penetrate into tumor cells and diffuse through the blood brain barrier; this makes drug 5FC a good choice for treating hard-to-reach tumors such as glioblastoma [37, 38]. With the introduction of high concentrations of cytotoxic molecules to the tumor environment, our current therapeutic index may be increased significantly while minimizing impact on normal tissues.

Despite significant successes already made in the field of suicide gene therapy, we should now optimize factors like transgene selection, delivery methods, enzyme engineering, transgene expression, and drug conversion rates.

\section{Conclusions}

Suicide gene therapy forces cancer cells to self-destruct while standard chemotherapy drugs damage both healthy and cancerous cells [5,35]. The therapeutic consequences of suicide gene therapy can be spread beyond the transfected tumor cells [5, 36]. As a result, distant tumor lesions can be regressed [5, 20]. Suicide gene therapy could be used as an additional cornerstone step in cancer treatment along with the conventional integrated multimodality approach of chemotherapy, radiotherapy and surgery [5]. This will help in the regression of tumors and possibly prevent multidrug resistance commonly observed in cancer patients [6, 10, 11]. IPSCs genetically engineered to express CD enzymes may be a valuable therapeutic option for treating cancer, but some hurdles must be overcome before they become a clinically efficient treatment of cancers. Possible solutions include vector design to enhance targeting and controlled delivery, combination of different suicide genes, more efficient activation of a given prodrug, and the creation of better substrates for the enzymes in order to maximize drug release and to allow the active drug to accumulate more readily in tumor cells.

Banks of iPSCs are very important to preserve their characteristic stemness and potency, to prevent contamination and deterioration, and to facilitate their effective use in basic and clinical applications [3, 21]. A number of stem cell banks have already been established in many countries, and their prevalence is expected to expand [3, 21]. Concurrently, there is still a substantial need for specialized therapeutic stem cells banks in order to determine the effectiveness of iPSCs in clinical applications. 


\section{Acknowledgements}

The authors want to thank experts working at Department of Radiology, Stanford University.

\section{Funding}

This work was supported by the Stanford University research fund of 2019 .

\section{Availability of data and materials}

Not applicable.

\section{Authors 'contributions}

MU: Conceptualization and writing of review manuscript.

\section{Ethics approval and consent to participate}

Not applicable.

\section{Consent for publication}

The article and the sources used have been cited in the article.

\section{Competing interests}

The author declare that he has no competing interests.

\section{Authors' information}

Interventional Regenerative Medicine and Imaging Laboratory, Stanford University School of Medicine, Department of Radiology, Palo Alto, California 94304, USA

\section{REFERENCES}

1. Siegel, R.L., K.D. Miller, and A. Jemal, Cancer statistics, 2019. CA Cancer J Clin, 2019. 69(1): p. 7-34.

2. Papaccio, F., et al., Concise Review: Cancer Cells, Cancer Stem Cells, and Mesenchymal Stem Cells: Influence in Cancer Development. Stem Cells Transl Med, 2017. 6(12): p. 2115-2125. 
3. Ullah, M., et al., iPS-derived MSCs from an expandable bank to deliver a prodrug-converting enzyme that limits growth and metastases of human breast cancers. Cell Death Discov, 2017. 3: p. 16064.

4. Eissenberg, L.G., et al., Suicide genes: monitoring cells in patients with a safety switch. Frontiers in Pharmacology, 2014. 5.

5. Karjoo, Z., X.G. Chen, and A. Hatefi, Progress and problems with the use of suicide genes for targeted cancer therapy. Advanced Drug Delivery Reviews, 2016. 99: p. 113-128.

6. Yi, B.R., S.U. Kim, and K.C. Choi, Synergistic effect of therapeutic stem cells expressing cytosine deaminase and interferon-beta via apoptotic pathway in the metastatic mouse model of breast cancer. Oncotarget, 2016. 7(5): p. 5985-99.

7. Marofi, F., et al., Mesenchymal Stromal/Stem Cells: A New Era in the Cell-Based Targeted Gene Therapy of Cancer. Front Immunol, 2017. 8: p. 1770.

8. Altanerova, U., et al., Prodrug suicide gene therapy for cancer targeted intracellular by mesenchymal stem cell exosomes. International Journal of Cancer, 2019. 144(4): p. 897-908.

9. Thomas, C.E., A. Ehrhardt, and M.A. Kay, Progress and problems with the use of viral vectors for gene therapy. Nature Reviews Genetics, 2003. 4(5): p. 346-358.

10. Ullah, M., et al., Mesenchymal stem cells confer chemoresistance in breast cancer via a CD9 dependent mechanism. Oncotarget, 2019. 10(37): p. 34353450.

11. Ullah, M., A. Akbar, and A.S. Thakor, An emerging role of CD9 in stemness and chemoresistance. Oncotarget, 2019. 10(40): p. 4000-4001.

12. Prockop, D.J., S.E. Prockop, and I. Bertoncello, Are clinical trials with mesenchymal stem/progenitor cells too far ahead of the science? Lessons from experimental hematology. Stem Cells, 2014. 32(12): p. 3055-61.

13. Zhao, Q., et al., MSCs derived from iPSCs with a modified protocol are tumortropic but have much less potential to promote tumors than bone marrow MSCs. Proc Natl Acad Sci U S A, 2015. 112(2): p. 530-5.

14. Melzer, C., Y. Yang, and R. Hass, Interaction of MSC with tumor cells. Cell Commun Signal, 2016. 14(1): p. 20.

15. Ullah, M., et al., Stem cell-derived extracellular vesicles: role in oncogenic processes, bioengineering potential, and technical challenges. Stem Cell Research \& Therapy, 2019. 10(1).

16. Bartosh, T.J., et al., Cancer cells enter dormancy after cannibalizing mesenchymal stem/stromal cells (MSCs). Proc Natl Acad Sci U S A, 2016. 113(42): p. E6447-E6456.

17. Ullah, M., et al., Emerging role of stem cell-derived extracellular microRNAs in age-associated human diseases and in different therapies of longevity. Ageing Res Rev, 2020. 57: p. 100979.

18. Jonnakuti, S.T. and M. Ullah, ANTI-AGING PROTEIN CD9 AFFECTS AGERELATED HEART FAILURE. Innovation in Aging, 2019. 3(Suppl 1): p. S255.

19. Harris, D.T., Stem Cell Banking for Regenerative and Personalized Medicine. Biomedicines, 2014. 2(1): p. 50-79. 
20. Sait, S.F. and M.A. Karajannis, The new kid on the block: suicide gene therapy to modulate cancer immunosurveillance for children with high-risk malignant brain tumors. Neuro-Oncology, 2019. 21(4): p. 419-420.

21. Sun, C., et al., Fundamental Principles of Stem Cell Banking. Adv Exp Med Biol, 2016. 951: p. 31-45.

22. Ullah, M., et al., iPS-derived MSCs from an expandable bank to deliver a prodrug-converting enzyme that limits growth and metastases of human breast cancers. Cell Death Discovery, 2017. 3.

23. Ullah, M., D.D. Liu, and A.S. Thakor, Mesenchymal Stromal Cell Homing: Mechanisms and Strategies for Improvement. iScience, 2019. 15: p. 421-438.

24. Shologu, N., et al., Adipose Derived Mesenchymal Stem Cell Therapy Is Comparable or Superior to Bone-Marrow Derived on Hypoxia-Induced Lung Injury in Primary Rat Alveolar Epithelial Cells. American Journal of Respiratory and Critical Care Medicine, 2018. 197.

25. Hwang, G., et al., Efficacies of Stem Cell Therapies for Functional Improvement of the beta Cell in Patients with Diabetes: A Systematic Review of Controlled Clinical Trials. Int J Stem Cells, 2019. 12(2): p. 195-205.

26. Laplane, L., et al., Concise Review: Induced Pluripotent Stem Cells as New Model Systems in Oncology. Stem Cells, 2015. 33(10): p. 2887-92.

27. Scudellari, M., How iPS cells changed the world. Nature, 2016. 534(7607): p. 310-2.

28. Takahashi, K. and S. Yamanaka, Induced pluripotent stem cells in medicine and biology. Development, 2013. 140(12): p. 2457-2461.

29. Pei, D.Q., et al., Induced Pluripotent Stem Cell Technology in Regenerative Medicine and Biology. Bioreactor Systems for Tissue Engineering li: Strategies for the Expanison and Directed Differentiation of Stem Cells, 2010. 123: p. 127141.

30. Guhr, A., et al., Recent Trends in Research with Human Pluripotent Stem Cells: Impact of Research and Use of Cell Lines in Experimental Research and Clinical Trials. Stem Cell Reports, 2018. 11(2): p. 485-496.

31. Kim, J.H., et al., Report of the International Stem Cell Banking Initiative Workshop Activity: Current Hurdles and Progress in Seed-Stock Banking of Human Pluripotent Stem Cells. Stem Cells Translational Medicine, 2017. 6(11): p. 1956-1962.

32. Ilic, D., et al., Human embryonic and induced pluripotent stem cells in clinical trials. British Medical Bulletin, 2015. 116(1): p. 19-27.

33. Ye, M., et al., Exosomes Derived from Human Induced Pluripotent Stem CellsEndothelia Cells Promotes Postnatal Angiogenesis in Mice Bearing Ischemic Limbs. International Journal of Biological Sciences, 2019. 15(1): p. 158-168.

34. Zarogoulidis, P., et al., Suicide Gene Therapy for Cancer - Current Strategies. J Genet Syndr Gene Ther, 2013. 4.

35. Kalimuthu, S., et al., Genetically engineered suicide gene in mesenchymal stem cells using a Tet-On system for anaplastic thyroid cancer. PLoS One, 2017. 12(7): p. e0181318. 
36. Hao, Y., et al., Proliferation inhibition and apoptosis enhancement of human cervical cancer cells by ultrasound-targeted microbubble destruction delivered double suicide genes. Int J Clin Exp Med, 2014. 7(12): p. 5330-5.

37. Young, J.S., et al., Advances in stem cells, induced pluripotent stem cells, and engineered cells: delivery vehicles for anti-glioma therapy. Expert Opin Drug Deliv, 2014. 11(11): p. 1733-46.

38. Yagiz, K., et al., A Retroviral Replicating Vector Encoding Cytosine Deaminase and 5-FC Induces Immune Memory in Metastatic Colorectal Cancer Models. Molecular Therapy-Oncolytics, 2018. 8: p. 14-26. 